\title{
Pedestrian evacuation based on an improved dynamic parameters model
}

\author{
Zhu Nuo ${ }^{1, *}$ Shao Fengru \\ ${ }^{1}$ China Academy of Civil Aviation Science and Technology, Beijing, 100028, China \\ *zhunu051@163.com
}

Keywords: cellular automata, pedestrian evacuation, dynamic parameter, evacuation time. Abstract: A improved dynamic parameters model is presented based on cellular automata in this paper.The dynamic parameters: Direction-parameter, Empty-parameter and Cognition-parameter are formulated to simplify tactically the decision-making process of pedestrians, which can reflect the pedestrian judgment on the surrounding conditions and decide the pedestrian's choice of action. Simulation of pedestrian evacuation and pedestrian moving rules were established, according to two-dimensional cellular automaton Moore neighborhood. In the improved model, the impact of the pedestrian density around exits is considered, the simulation and experimental results prove that this improvement makes sense, because besides the spatial distance to exits, people may also choose the exit according to the pedestrian density around exits. And the simulation results of improved model is compared with experiment, comparison shows that the improved model can reproduce the experiment well. The improved model is useful for further study, it is instructional significant for pedestrian evacuation, avoiding or reducing the number injuries.

\section{Introduction}

Based on the characteristics of individual pedestrian and system surroundings, the traditional simulation models are extended, combined or modified to approximate pedestrian dynamics[1-4]. A vector-based particles field is introduced in the Floors Field Model [5] to represent crowd forces between pedestrians in pedestrian evacuation simulation. The Multi-grid Model [6] introduces the force concept of the Social Force Model into the Lattice Gas Model. A modified CA model [7] which involves the Floor Field Model and the Social Force Model simulate pedestrian evacuation with obstacles and multi-exits. In most pedestrian evacuation simulations [8], the room is supposed to be a rectangle box with one exit. However, rooms with more than one exit can be frequently observed in realistic life, and the exits layout will affect the pedestrian evacuation process. Pedestrians' exit selection strategy will be a basic rule in the evacuation simulation. And it is well accepted that rational pedestrians are intelligent and adaptive to the dynamic conditions around by constantly seeking and choosing an optimum route. So pedestrians who are familiar with evacuation circumstances without panics will intend to leave room within the shortest possible time. In the previous models [9-11], crowd distribution is not considered or it is supposed that the crowd is uniformly distributed in a large room without any obstacle, but this parameter is important in specifying doors locations. In this paper, the dynamic parameters model is presented to simulate pedestrian multiexit evacuation, which can also be adapted to the simulation with one exit.

\section{Model description}

The model based on the previous models [10-11], proposed on $(W+2) \times(W+2)$ two-dimensional discrete grid, where the movement area in the system is marked out into $W \times W$ cells and $W$ is the system size. Discrete cellular has the same size, barriers occupy the boundary's cellular and consist of the wall, some empty cellular left on the wall as an exit (see Fig. 1(a)). In each discrete time step, pedestrian can choose to wait or move to the next possible position (see Fig. 1(b)). The transition payoff (a $3 \times 3$ matrix) is introduced to describe the relative advantages to be obtained by a certain pedestrian (see Fig. 1(c)) through Direction-parameter, Empty-parameter and Cognition-parameter. 
Once the geometry of the system is determined, each cell is assigned a constant value representing its distance to the door. The distance indicates the degree of attractiveness of a cell to pedestrians:

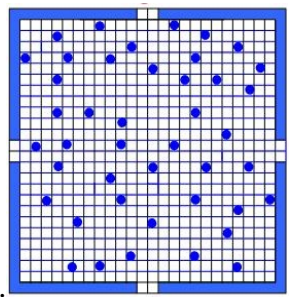

(a) Simulation system

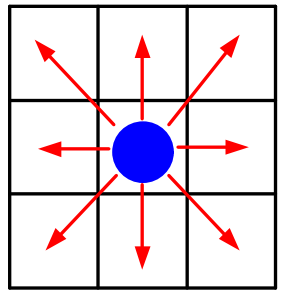

(b)The movement field.

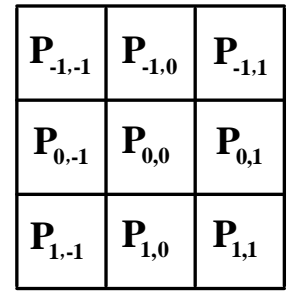

(c)The matrix of transition payoffs

Fig.1 Simulation model of evacuation pedestrian

Direction-parameter indicates the cell's degree of approximation to the pedestrian destination, namely room exit with a single step in a time step. The values of elements of Direction-parameter matrix are given as:

$$
D_{i j}=\frac{S_{00}-S_{i j}}{\sqrt{\mathrm{i}^{2}+j^{2}}}
$$

Where $S_{00}$-the shortest distance from the core cell in the movement field to the evacuation exits. $S_{i j}$-the shortest distance from the cell $(i, j)$ in the movement field to the evacuation exits.

Empty-parameter indicates whether the cell is occupied or not. The values of elements of Empty-parameter matrix are given as

$$
E_{i j}=\left\{\begin{array}{lr}
\max \left(D_{i j}\right), & \text { empty cells, } \\
0, & \text { the core cell, } \\
-\max \left(D_{i j}\right), & \text { occupy cells, }
\end{array}\right.
$$

Cognition-parameter describes the effects of pedestrian jam around exits and the width of exits. The values of elements of Cognition-parameter matrix are given as:

$$
C_{i j}=\max \left(\max _{m}\left(\frac{d_{m} \cdot\left(p_{\text {in }}^{t} / S_{i n}^{\mathrm{t}}\right.}{d_{L} \cdot\left(p_{m}^{t} / S_{m}^{\mathrm{t}}\right)}\right)\right)
$$

Where $d_{m}$-the width of $m$-th exits; $d_{L}$-the sum width of exits; $p_{\text {in }}^{t}$-the number of pedestrians in the evacuation system at $t$ time step; $p_{\mathrm{m}}^{t}$-the number of pedestrians around $m$-th exits at $t$ time step; $S_{i n}^{\mathrm{t}}$-the region of the evacuation system at $t$ time step; $S_{m}^{\mathrm{t}}$-the evacuation region around $m$-th exits at $t$ time step .

\section{Rules}

In our model, a set of intelligent local rules are introduced into our model:

(1) In the model, pedestrian has nine possible target positions and only one of them can be selected in each time step.

(2) Define transition payoff $\mathrm{P}_{i j}$ as:

$$
P_{i j}=\alpha \cdot D_{i j}+\beta \cdot E_{i j}+\gamma \cdot C_{i j}
$$

Where $\alpha+\beta+\gamma=1$, however, we strongly recommended that $\beta \geq \alpha+\gamma$, because once the target cell is occupied, even if Direction-parameter and Cognition-parameter is big enough, the pedestrian will not enter this cell. The pedestrian would choose the cell with the largest value $\mathrm{P}_{M}$ in the matrix of transition payoff as his or her target position. $\mathrm{P}_{M}=\max \left(\mathrm{P}_{i j}\right)$.

(3) If more than one possible target cells rank the highest in value $\mathrm{P}_{M}$, only one of them will be 
chosen as the target position randomly with equal probability.

(4) A conflict occurs when any two or more pedestrians attempt to move to the same target position. In this situation, only one of them will be chosen randomly with equal probability.

(5) When a pedestrian moves into the exit cell, at the next time step the pedestrian will leave the room system. After leaving the room, the pedestrian would not reenter the room system.

(6) When all the pedestrians leave the room and there is no pedestrian in the evacuation room, the simulation procedure is terminated.

\section{Simulation analysis}

As mentioned above, simulations are performed according to the updating rules, our model has considered the influence of pedestrian density compared with the previous model, and combined it with other parameters by bringing in a new parameter $C_{i j}$ and its impact factor $\gamma$. The relationships between impact factors $\beta, \gamma$ and evacuation time are plotted in Fig. 2(a). We plot the difference between experimental and simulation results versus pedestrian density in Fig.(b),we find that when $\beta=0.5, \gamma=0.35$, the simulation results agree with the experimental results best.
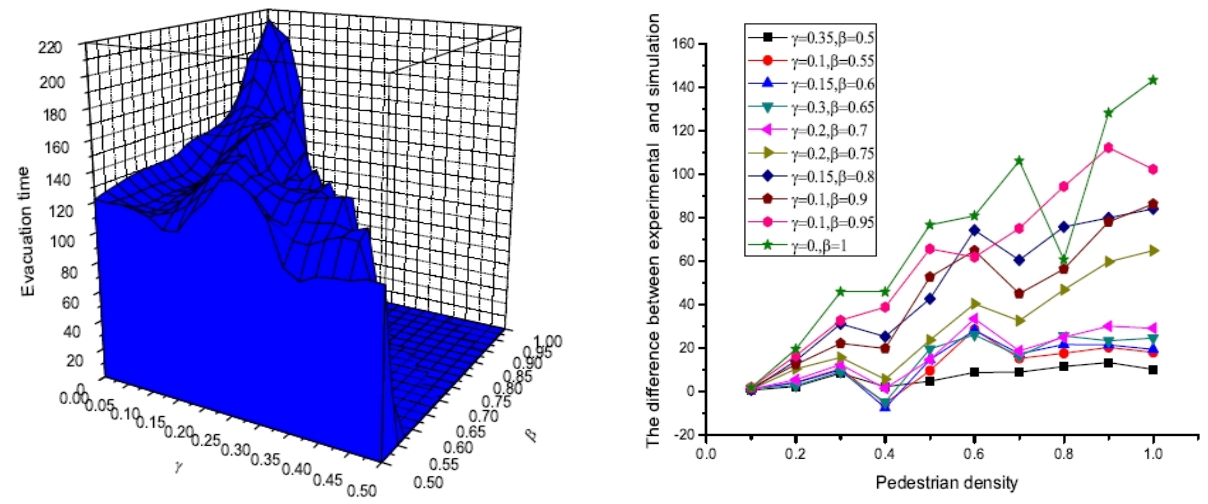

(a)The curve of evacuation time versus $\beta$ and $\gamma$

(b) the difference between experimental and simulation

Fig. 2. The influence of parameter variation

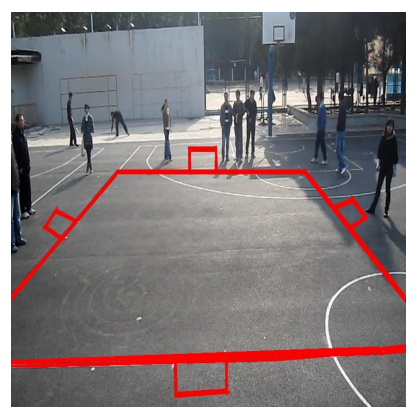

(a)

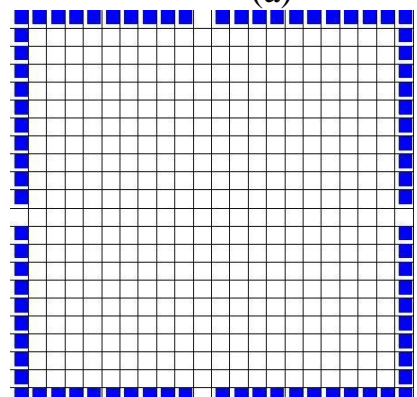

(e)

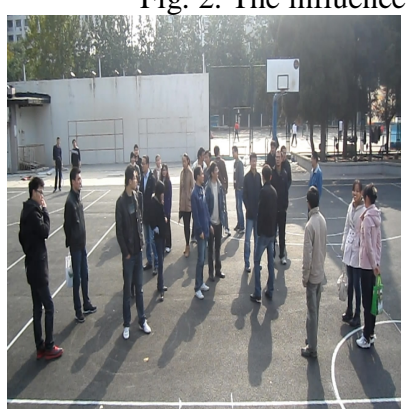

(b)

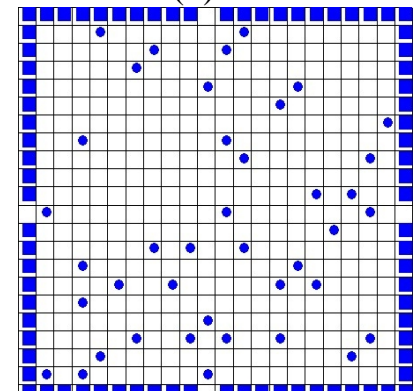

(f)

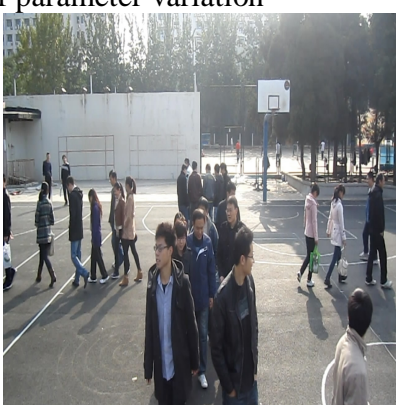

(c)

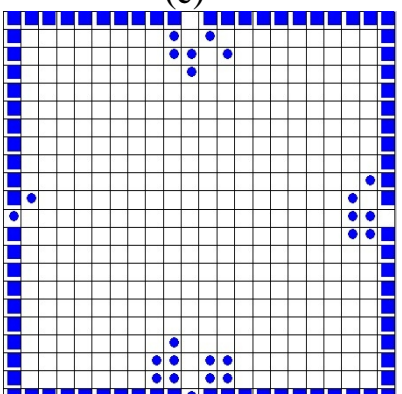

(g)

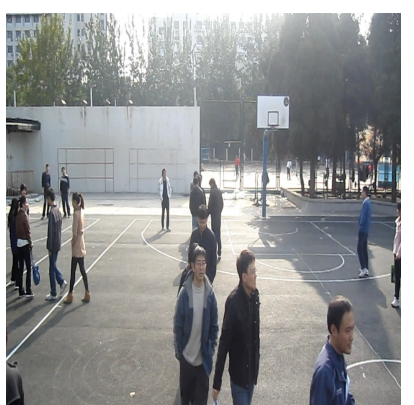

(d)

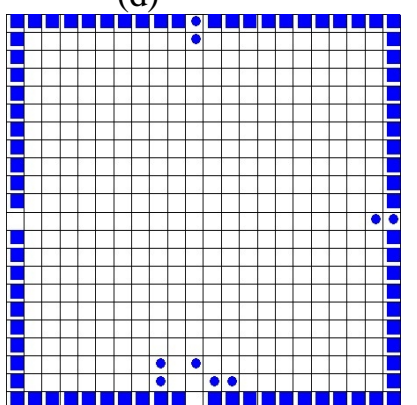

(h)

Fig3 (a) - (d) Actual Pedestrian Movement; (e) - (h) the Process of Simulation

In order to show the superiority of our models, we do some experiments which are carried out in a room (imaginary room in the playground) with an area of $8 \mathrm{mX8m}$ (see Fig.3(a)). There are four doors in the room at the center of each wall, the width of each door is $0.5 \mathrm{~m}$. The evacuation processis recorded by four cameras fixed at the top of doors, some students with ages of 20-25 years take part in 
the experiment, so individual differences are not significant. Fig. 3(b)-(d) show the actual process of evacuation. At the same time, we simulate the snapshots of evacuation process in this environment (see Fig.3(e)-(h)). We can see our model's simulation results are reasonable and the simulation results agree with the experiment results quite well.

\section{Conclusions}

In this paper, a dynamic parameters model is established based on cellular automata.The dynamic parameters: Direction-parameter, Empty-parameter and Cognition-parameter are formulated to simplify tactically the decision-making process of pedestrians, which can reflect the pedestrian judgment on the surrounding conditions and decide the pedestrian's choice of action. Simulation of pedestrian evacuation is established, according to two-dimensional cellular automaton Moore neighborhood. Through simulation results and experiment we set the optimal value of impact factor. The research in this paper reminds us that the pedestrian density around exit is also playing an important role in pedestrian evacuation beside space distance, the improved model can produce a more realistic outcome by considering the exit pedestrian density. Research objective is to identify information that might be useful in building designing to assess buildings and their ability to provide sufficient time for the pedestrians to evacuate safely in the event of a emergency.

\section{Acknowledgements}

This work is financially supported by the National Natural Science Foundation of China (71071013, 71001004, and 71071012).

\section{References}

[1] C. Burstedde, K. Klauck, A. Schadschneider, J. Zittartz, Simulation of pedestrian dynamics using a two-dimensional cellular automaton, Physica A 295 (2001) 507-525.

[2] D. Helbing, P. Molnar, Social force model for pedestrian dynamics, Phys. Rev.E 51 (5) (1995) $4282-4286$.

[3] M. Muramatsu, T. Irie, T. Nagatani, Jamming transition in pedestrian counter flow, Physica A 267 (1999) 487-498.

[4] D. Helbing, I. Farkas, T. Vicsek, Simulating dynamical features of escape panic, Nature 407 (2000) 487-490.

[5] C.M. Henein, T.White, Macroscopic e ${ }^{\circledR e c t s}$ of microscopic forces between agents in crowd models, Physica A 373 (2007) 694-712.

[6] W. Song, X. Xu, B.Wang, S. Ni, Simulation of evacuation processes using a multi-grid model for pedestrian dynamics, Physica A 363 (2006) 492-500.

[7] S. Liu, L. Yang, T. Fang, J. Li, Evacuation from a classroom considering the occupant density around exits, Physica A 388 (2009) 1921-1928.

[8] W. Yuan, K. Tan, An evacuation model using cellular automata, Physica A 384(2007) 549-566.

[9] H. Yue, C. Shao, X. Chen, H. Hao, Simulation of bi-directional pedestrian flow based on cellular automata model, Acta Phys. Sinica 57 (2008) 6901-6908.

[10] Zhu Nuo, Jia Bin, Shao Chun-fu, Yue Hao. Simulation of pedestrian evacuation based on an improved dynamic parameter model, Chinese Physics B.21(5) ( 2012). 050501.

[11]Zhu Nuo, Jia Bin, Shao Chun-fu. Pedestrian evacuation based on a dynamic parameters model. Applied Mechanics and Materials, 97-98(2011) 956-959 